\title{
Archéopages
}

Archéopages

Archéologie et société

$43 \mid 2016$

Médecines

\section{Organisation médicale pendant la première guerre mondiale}

L'exemple de l'hôpital américain de Saint-Parize-le-Châtel

Medical organization during the First World War. The example of the American

hospital in Saint-Parize-le-Châtel

\section{Nicolas Tisserand et Alexandre Coulaud}

\section{OpenEdition}

Journals

Édition électronique

URL : https://journals.openedition.org/archeopages/2241

DOI : 10.4000/archeopages.2241

ISSN : 2269-9872

Éditeur

INRAP - Institut national de recherches archéologiques préventives

Édition imprimée

Date de publication : 1 novembre 2016

Pagination : 68-71

ISSN : 1622-8545

\section{Référence électronique}

Nicolas Tisserand et Alexandre Coulaud, «Organisation médicale pendant la première querre mondiale », Archéopages [En ligne], 43 | 2016, mis en ligne le 01 décembre 2018, consulté le 06 juin 2021. URL : http://journals.openedition.org/archeopages/2241 ; DOl : https://doi.org/10.4000/ archeopages. 2241 


\section{Organisation médicale \\ pendant la première guerre mondiale}

L'exemple de l'hôpital américain de Saint-Parize-le-Châtel

Nicolas Tisserand Irrap, UMR 629\& «A A TERHiS"

Alexandre Coulaud Irrap

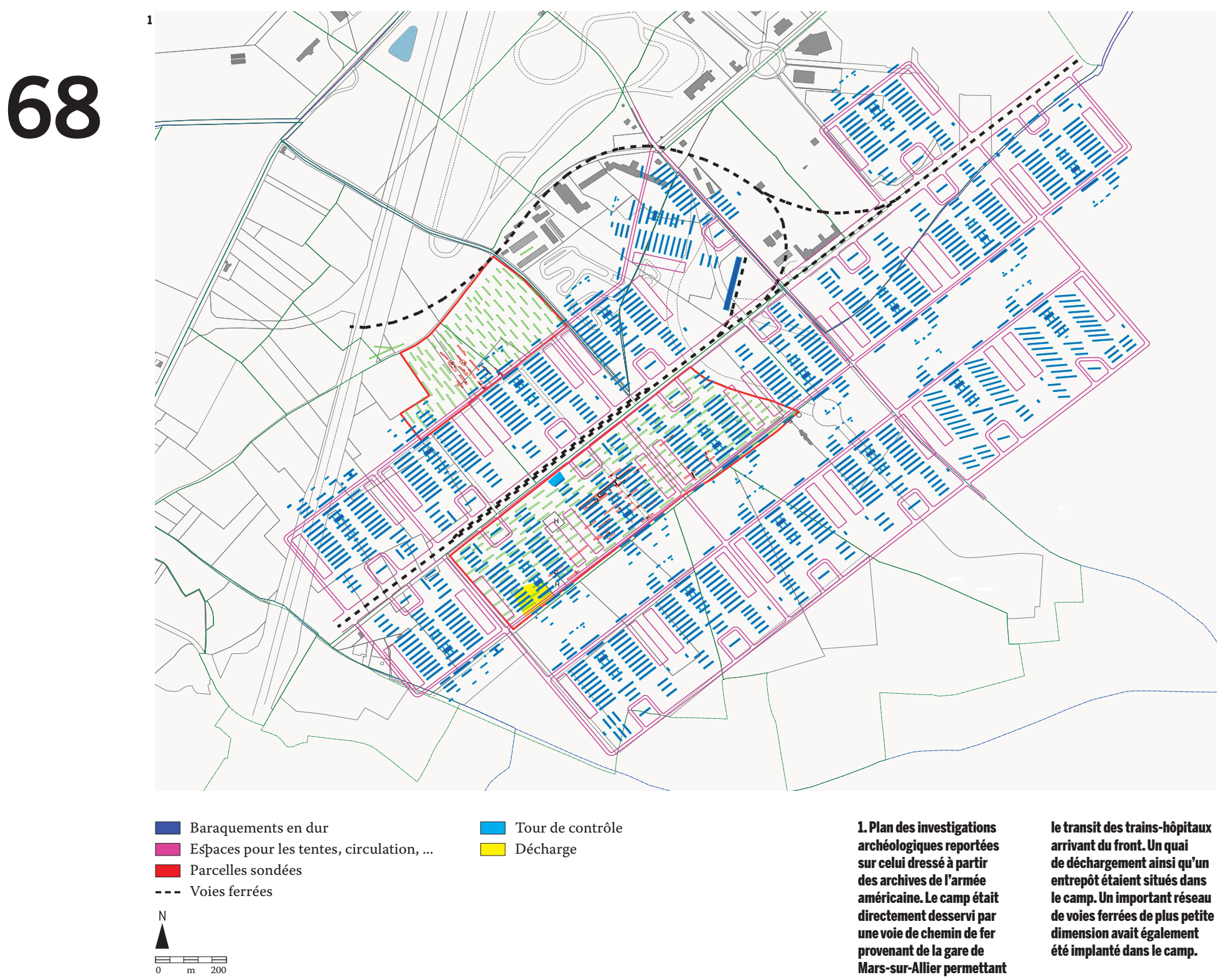


Un diagnostic réalisé en 2014 à Saint-Parizele-Châtel (Nièvre) ${ }^{\mathbf{1}}$ a permis la redécouverte de vestiges liés à un hôpital américain datant de la première guerre mondiale (Tisserand, Coulaud, 2014) [ill. 1]. Outre des maçonneries bien visibles dans le paysage jusqu'il y a peu et ceux dégagés en 2014, nous disposons d'un fonds d'archives exceptionnel constitué de nombreuses photographies commentées, de plans et croquis du camp, de notes sur la vie quotidienne. La vigilance des acteurs et bénévoles locaux a également permis de constituer une collection d'objets médicaux ou personnels provenant de ramassage sur site, de récupération et de dons. Les vestiges mis au jour ne concernaient qu'une petite partie de cet hôpital, mais la relative normalisation de ces constructions, la présence de vestiges encore en élévation ainsi que cette très riche documentation ont permis de travailler sur l'ensemble de l'hôpital.

\section{Le contexte médical : l'expérience américaine et les innovations françaises}

Lorsque, le 6 avril 1917, le président Wilson officialise l'entrée en guerre des États-Unis d'Amérique contre l'Allemagne, un vaste effort de création d'infrastructures médicales est engagé. L'American Expeditionary Force (AEF) met en place de nombreux hôpitaux et des services d'évacuation sanitaire. Ainsi, ce sont près d'une cinquantaine d'hôpitaux américains de campagne qui sont établis sur le territoire national, dont celui de Saint-Parize-le-Châtel communément appelé «Mars-sur-Allier base hospital» du nom de la gare régulatrice où étaient amenés les blessés, située à quelques kilomètres. L'armée américaine bénéficie d'un service de santé relativement organisé et efficace et ce, grâce aux enseignements tirés des ravages de la guerre de Sécession (1861-1865) qui fit plus de 600 ooo morts et 500 ooo blessés. En sus des avancées chirurgicales en lien avec les nouveaux types de blessures, cela eut pour conséquence, notamment, de regrouper les malades par catégories et de développer des champs de spécialisation.

La première guerre mondiale voit également le développement de la radiographie médicale mobile et ainsi, à l'initiative de Marie Curie et d'Antoine Beclère, de nombreux spécialistes, dont une vingtaine d'Américains, sont formés et plus de deux cents salles de radiologie installées dans les hôpitaux des armées. Les équipements américains sont à la pointe du progrès technologique et la radiographie est aussitôt utilisée par les médecins de l'AEF. Cela se matérialise notamment par la présence d'un local de radiographie au moins dans un des baraquements d'une des «base hospital $\left(n^{\circ} 48\right) »$ du camp de Saint-Parize.

\section{L'organisation du camp}

Pendant l'hiver 1917-1918, c'est une véritable ville qui est construite sur les pentes à l'ouest du village de Saint-Parize-le-Châtel. Un important contingent de soldats indochinois provenant de l'empire colonial français sont mis à disposition de l'AEF pour la construction et l'entretien du camp. Le camp américain $\mathrm{APO} 780$, entièrement dédié au domaine sanitaire, est actif pendant les derniers mois de 1918 et les premiers de $1919^{2}$, soit 171 jours. Sur près de 330 ha, les 700 constructions en dur étaient destinées à accueillir 20 ooo lits permanents ; 20 ooo lits supplémentaires pouvaient être dressés sous tentes en cas de crises majeures. Les deux camps de convalescence comptaient 5 ooo places chacun. Le camp regroupait la pointe des équipements médicaux modernes : blocs opératoires, salles de radiologie, de nombreux laboratoires (homéopathie, chimie, bactériologie...) [ill. 2].

De nombreux malades de la grippe espagnole, qui frappa les forces armées américaines, furent pris en charge et l'hôpital accueillit également des soldats allemands et français blessés. On estime à environ 37774 le nombre de patients qui passèrent par les services de l'hôpital : 12599 y reçurent des soins jusqu'en convalescence, mais 438 y décédèrent ; 332 opérations furent réalisées (dont 6 sur des prisonniers allemands) et 1148 radiographies.

Le personnel de cet hôpital géant, qui n'a cependant jamais fonctionné à plein régime, comptait plusieurs milliers de personnes sans que l'on sache précisément combien. Pour la «base hospital $\left(\mathrm{n}^{\circ} 48\right)$ » nous savons que le personnel comptait 50 officiers (médecins notamment), 100 infirmières et 238 soldats engagés volontairement dans l'armée américaine pour servir de brancardiers, d'infirmiers ou d'aides dans les hôpitaux ou les centres logistiques, ainsi que 6 civils. L'hôpital était subdivisé en 20 « base hospital » de type A, chacun établi sur le même modèle, disposant des mêmes services et séparé des autres par des clôtures gardées. Le camp disposait de son propre réseau électrique, d'eau courante, d'une chapelle, d'un cimetière, mais également d'une salle de spectacle, d'un orchestre, d'une équipe de football et même d'un petit journal hebdomadaire, The Martian, édité jusqu'au 27 avril 1919.

En 1919, à l'abandon du camp, neuf blocs étaient des «base hospital» de type A, c'est-à-dire des hôpitaux de campagne sous forme de baraquements. Sur les onze autres blocs, un, situé à l'entrée du camp, était consacré au transport (logistique, médical...), un était réservé aux civils (des soins à la population étaient dispensés comme dans tous les autres hôpitaux militaires durant le conflit), les autres n'ayant jamais été occupés. Le camp comprenait d'autres aménagements annexes ne figurant pas sur le plan de l'AEF. Sur la partie sommitale du camp, au sud-est, étaient établis les deux camps de convalescence. Dans le même secteur, on trouvait le quartier général du camp ainsi que deux réservoirs semi- 
enterrés destinés à alimenter le camp en eau (remplacés par un château d'eau, encore en élévation, mais qui n'a jamais été mis en service).

\section{La «base hospital type A »}

Chaque base est établie sur un plan similaire rectangulaire de 274 X 244 $\mathrm{m}$ [ill. 3]. Le plan d'une base est caractérisé par cinq zones distinctes séparées par des voies de communication. Ces divisions répondent à des critères d'ordres divers : patient/soignant ; homme/femme ; officiers/ soldats ; zones de vie/zones de soin/zones techniques. Notons l'intérêt porté à l'hygiène, manifesté par la présence d'un bâtiment dédié à la désinfection (ustensiles médicaux, linges...), de salles de bains, d'une buanderie et d'un incinérateur à déchets (médicaux et communs). Chaque base, disposant de l'eau courante, de l'électricité, du téléphone et de l'évacuation des eaux usées, était prévue pour un fonctionnement quasi autonome (garage, stockage, cuisine, foyer, pharmacie...) et possédait son quartier général qui dominait la place d'armes et le mât des couleurs.

\section{Le mobilier}

Le camp étant administré par des personnels américains, le mobilier importé ou apporté des États-Unis est omniprésent et ce quel que soit son usage : du parfum venant de New York, des bouteilles de lait en verre de Racine, des thermomètres de Rochester. Ce mobilier a été mis au jour soit à proximité des baraquements, soit dans des fosses dépotoirs [ill. 4a et b]. On retrouve en premier lieu des matériaux de construction (briques, clous, toile goudronnée). L'ensemble des objets de la vie quotidienne est également abondant, principalement des fragments de vaisselle en provenance de divers États américains, et des couverts, généralement siglés au nom de l'US Army. Parmi les objets en lien avec le soin ou l'hygiène ont été retrouvés de très nombreux flacons et autres contenants à usage médical et des instruments médicaux (seringues, thermomètre), mais également des rasoirs, des bouteilles de parfums, des poires à poudre, des brosses à dents. Concernant ces dernières, les modèles fabriqués en France sont en os alors que ceux provenant des États-Unis sont en plastique. L'embargo sur l'ivoire durant la guerre de Sécession y avait promu l'utilisation de ce matériau moderne. Des éléments d'habillement ont également été découverts (boutons, épingles, baleines). Les vestiges en lien avec l'alimentation sont moins importants en nombre. Il s'agit de boîtes de conserve en métal et de restes carnés, suffisamment nombreux pour laisser penser que la consommation de viande bovine était importante. Les pièces de viandes sélectionnées sont plutôt de très bonne qualité, issues d'animaux abattus à maturité.

Plan rationalisé, hygiène, innovations médicales, mobilier importé quasi en intégralité... Le camp militaire américain fut aussi une des premières images concrètes de la culture américaine découverte par les Français.

4. a

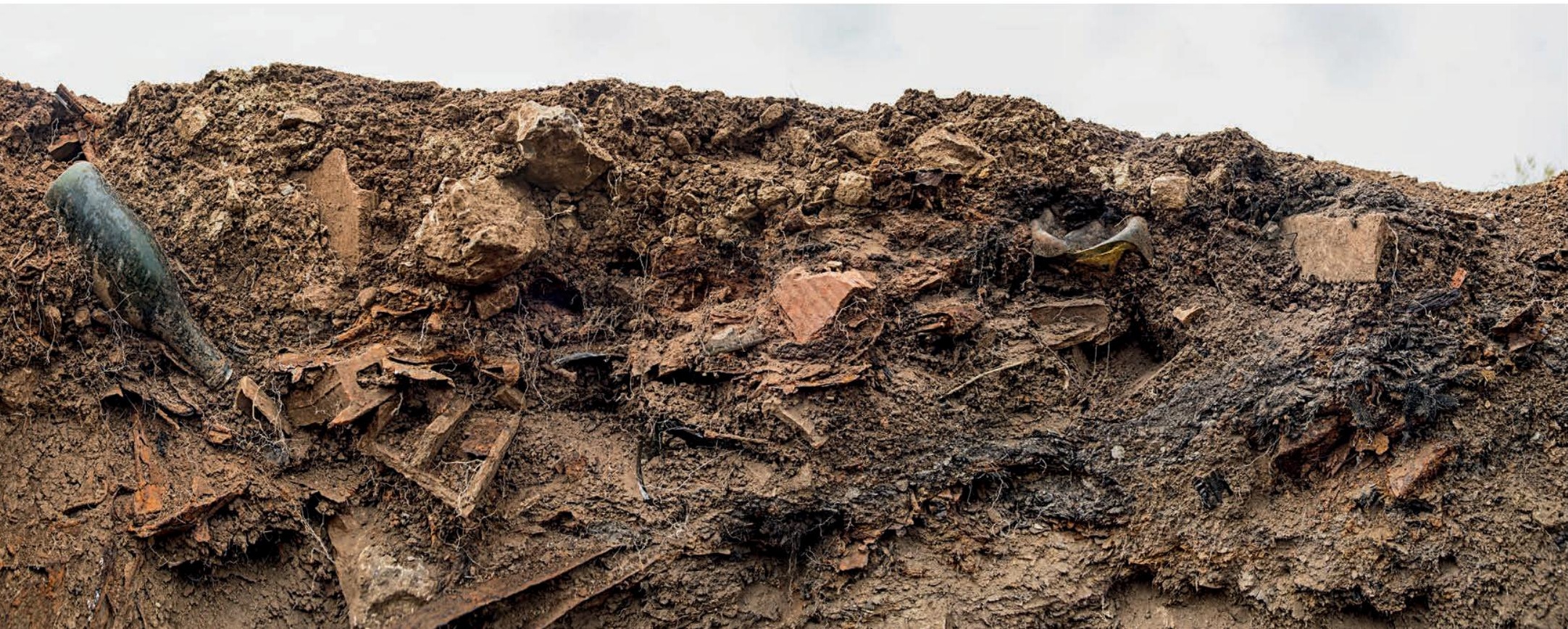


2. Laboratoire de préparation.

C'est dans un de ces

laboratoires que le chimiste

britannique Henry Drysdale

Dakin et le chirurgien français

Alexis Carrel inventèrent la

liqueur de Dakin, permettant

de réelles avancées dans

le traitement des plaies

ouvertes importantes et

infectées.
3.Plan détaillé de la « base hospital 48 » établi par

le sergent-chef Mort Pringle. 4. a et b. Coupe et fouille d'une des fosses dépotoirs,

deux d'entre elles ayant été partiellement documentées lors du diagnostic.
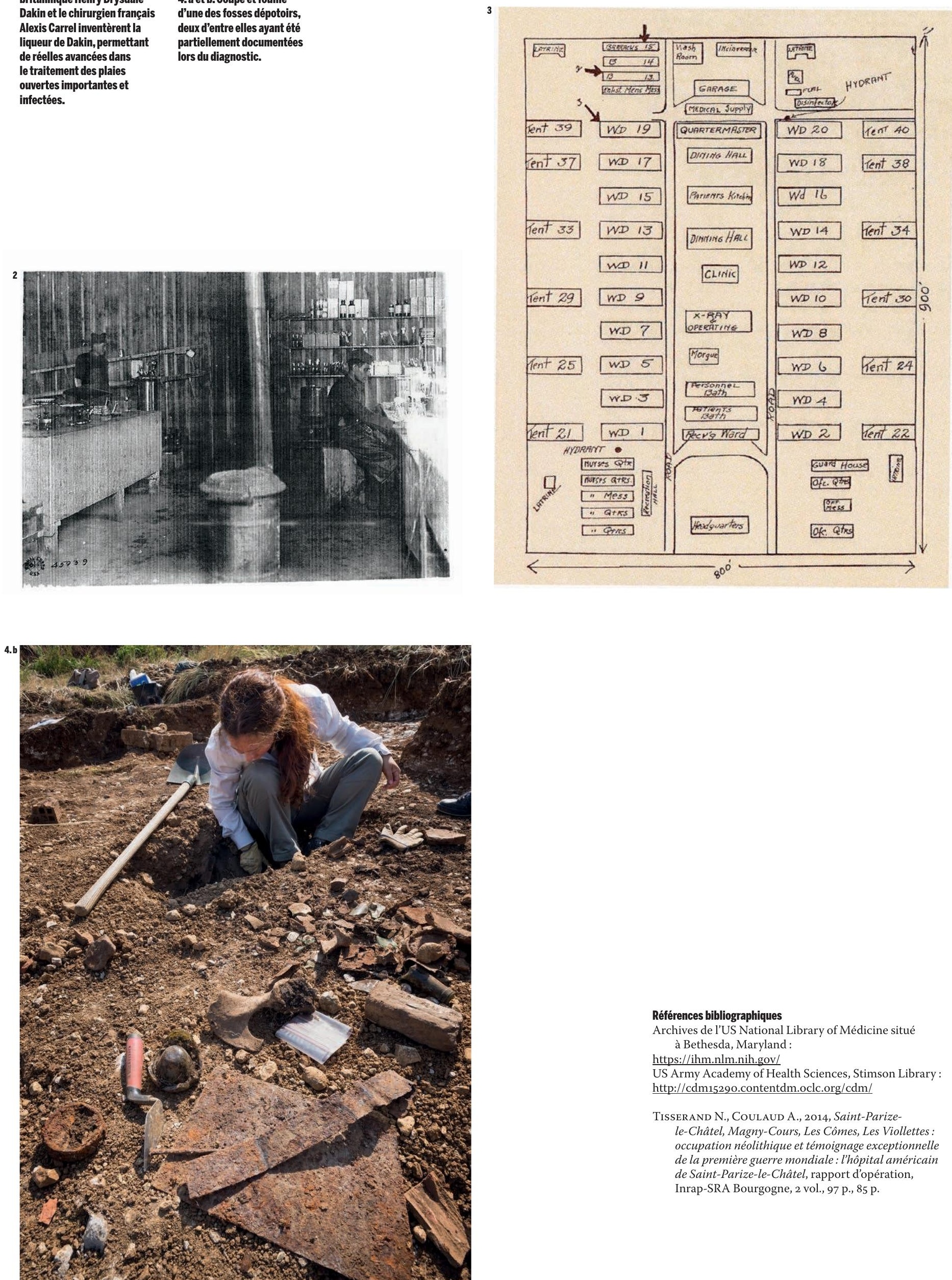

\section{Références bibliographiques}

Archives de l'US National Library of Médicine situé à Bethesda, Maryland :

https://ihm.nlm.nih.gov/

US Army Academy of Health Sciences, Stimson Library http://cdm15290.contentdm.oclc.org/cdm/

Tisserand N., Coulaud A., 2014, Saint-Parizele-Châtel, Magny-Cours, Les Cômes, Les Viollettes : occupation néolithique et témoignage exceptionnelle de la première guerre mondiale : l'hôpital américain de Saint-Parize-le-Châtel, rapport d'opération, Inrap-SRA Bourgogne, 2 vol., 97 p., 85 p. 